\title{
Highly efficient solution-processed white organic light-emitting diodes based on novel copolymer single layer
}

\author{
Carmen Coya ${ }^{\mathrm{a}, *}$, Angel Luis Álvarez ${ }^{\mathrm{a}}$, Mar Ramos ${ }^{\mathrm{b}}$, Rafael Gómez $^{\mathrm{c}}$, Carlos Seoane $^{\mathrm{c}}$, José Luis Segurac \\ a Departamento de Tecnología Electrónica, Escuela Superior de Ciencias Experimentales y Tecnología, Universidad Rey Juan Carlos, E-28933 Madrid, Spain \\ b Departamento de Tecnología Química y Ambiental, Escuela Superior de Ciencias Experimentales y Tecnología, Universidad Rey Juan Carlos, E-28933 Madrid, Spain \\ ' Departamento de Química Orgánica, Facultad de Ciencias Químicas, Universidad Complutense de Madrid, E-28040 Madrid, Spain
}

\section{A R T I C L E I N F O}

\section{Article history:}

Received 9 May 2011

Received in revised form 18 July 2011

Accepted 2 August 2011

Available online 7 September 2011

\section{Keywords:}

Solid state lighting

WOLEDS

Solution processed

Charge transport

\begin{abstract}
A B S T R A C T
We fabricate by solution processed methods organic light emitting diodes with single-layer structure (ITO/(PEDOT:PSS)/co-polymer/Ba/Al) and study the transport properties of the final devices. The copolymer is novel poly(fluorene-alt-phenylene) (PFP) derivatives containing co-monomers, involving red-emitting 1,8-naphthalimide units as pendant groups $(0.0005,0.005,0.02$ and 0.08 wt\%) covalently attached. All the devices exhibited emission at very low driving currents in the $\mu \mathrm{A}$ range $(47-73 \mu \mathrm{A})$. White emission with luminous efficiency of $9.42 \mathrm{Cd} / \mathrm{A}$ at $50 \mu \mathrm{A}$ is obtained for the co-polymer with the smallest amount of chromophore. Commission Internationale de L'Eclairage (CIE) coordinates evolve from almost pure white color $(0.26,0.30)$ for low currents to stable cool white $(0.21,0.23)$. Increasing naphthalimide contents leads to stable green and orange emission with 3.07, 19.5 and $6.7 \mathrm{Cd} / \mathrm{A}$ efficiencies. The current-voltage response of the devices is analyzed by means of a numerical model that includes an injection mechanism based in the microscopic hopping theory and a field-dependent carrier mobility for the bulk transport regime. The fitting results allow to estimate the dependence of carrier mobility on polymer composition in the diodes.
\end{abstract}

(C) 2011 Elsevier B.V. All rights reserved.

\section{Introduction}

White organic light emitting diodes (WOLEDs) have been recently considered one of the top 10 technologies of the decade [1] due to their potential ability for energy saving, and the key properties of lightness, optimum heat dissipation and flexibility, seeking to replace conventional white light sources by means of large area panels. Since 2008, WOLED designed lamps are already available [2] and, in the near future, organic solid-state lighting is expected to move from decorative applications to technical lighting and general illumination. This will however require higher efficiency, color purity and lifetime, as well as improvements of processed materials and architectures to reduce production costs.

Since white light emitting polymers are solution-processed, they have become a strategic issue within the solid-state lighting (SSL) context [3]. Currently, white polymer light emitting diode (WPLED) technology is mainly focused in reducing manufacturing costs as well as to improve procedures for mass-production lines. In this context, solution-processed methods are receiving much attention due to their compatibility with profitable manufactur-

* Corresponding author. Tel.: +34 916647470.

E-mail address: carmen.coya@urjc.es (C. Coya). ing techniques for large area production, such as roll-to-roll (R2R). In fact, the old dichotomy between molecules and polymers has been replaced by evaporated or solution processed capability of the materials. WPLED efficiency is still a major concern, achieving so far lower scores than those obtained by phosphorescent materials [4], or molecule-based active layers deposited by evaporation [2]. Currently high efficiency values in WPLEDs are considered to be around a few Cd/A [5]. Consequently there is currently a significant need for new organic semiconducting materials that combine the ability to perform good processability and stability with efficient charge transport and light emission.

Recently, we have demonstrated tunable fluorescence emission from novel poly(fluorene-alt-phenylene) (PFP) derivatives containing co-monomers, involving red-emitting 1,8-naphthalimide units as pendant groups $(0.0005,0.005,0.02$ and $0.08 \mathrm{wt} \%)$ [6] covalently attached. The integration of chromophore within the polymer structure avoids phase separation and formation of aggregates. In spite of the naphthalimide content all polymers showed similar electrochemical properties, with HOMO-LUMO levels of -4.93 and $-2.1 \mathrm{eV}$ respectively. However, variation of naphthalimide chromophore concentration in the polymers induces significant changes in the current-voltage $(\mathrm{I}-\mathrm{V})$ response of the devices based on these materials. In this work, we have fabricated solution processed WPLEDs with single-layer structure (ITO/(PEDOT:PSS)/co-polymer/Ba/Al) to study the effect of the 


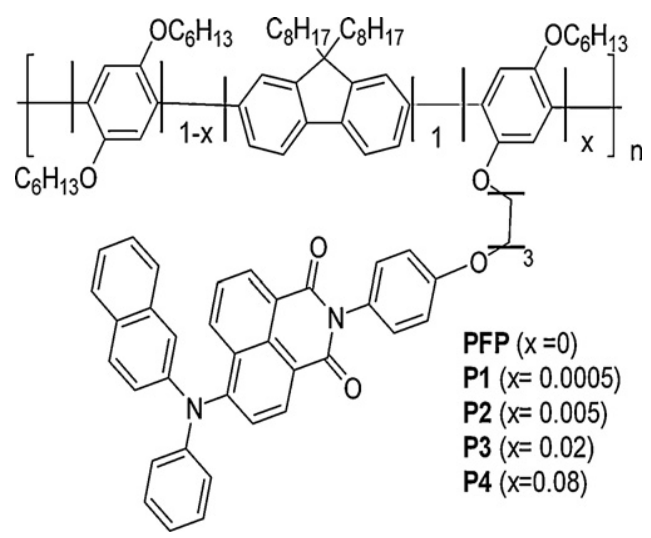

Fig. 1. Chemical structure of co-polymers P1-P4.

naphthalimide amount on the device properties, that is, the electroluminescence emission (EL) and transport properties. For this purpose, we use a numerical model that includes field-dependent carrier mobility to analyze the devices I-V responses, which allows us to estimate the carrier mobility of the novel copolymers.

\section{Material and experimental}

We use a blue-emitting poly(fluorine-alt-phenylene) (PFP) containing co-monomers bearing red-emitting 1,8-naphtalimide units as pendant groups. The synthetic route is reported elsewhere [4]. The naphtalimide dopant has been covalently attached to the pendant chain of the host with an alkyl spacer. See structure of the material in Fig. 1.

\subsection{Devices fabrication}

Pre-patterned ITO glass plates were extensively cleaned, using chemical and UV-Ozone methods, just before the deposition of the organic layers. A hole transport layer, PEDOT:PSS (from Baytron), is spin-cast at $6000 \mathrm{rpm}$ an dried at $110^{\circ} \mathrm{C}$ for $30 \mathrm{~min}$. The measured thickness of this layer was $75 \mathrm{~nm}$. The thicknesses were measured using an Alpha-step IQ profilometer (Tenkor Instruments). The active layer was then deposited using chloroform $\left(\mathrm{CHCl}_{3}\right)$ as a solvent in the precursor solution. Precursor solutions $(10 \mathrm{mg} / \mathrm{ml})$ were kept in an ultrasonic bath for $10 \mathrm{~min}$ and filtered through a $0.2 \mu \mathrm{m}$ polytetrafluoroethylene (PTFE) syringe filter, prior to be spin coated at $6000 \mathrm{rpm}$ for $45 \mathrm{~s}$, and then cured for $30 \mathrm{~min}$ at $50{ }^{\circ} \mathrm{C}$ on a hot plate to ensure solvent evaporation. The resulting layers thicknesses were: $78 \mathrm{~nm}, 163 \mathrm{~nm}, 99 \mathrm{~nm}$ and $121 \mathrm{~nm}$ for P1, P2, P3 and $\mathbf{P 4}$ respectively (Fig. 1). We have found that heating the sample just below the solvent boiling point reduces aggregation and results in an improved film uniformity (highly reflecting surfaces) [7]. The Al cathode was thermally evaporated in an atmosphere of $5 \times 10^{-5}$ mbar, after a thin layer of $\mathrm{Ba}$ (few $\mathrm{nm}$ ), on top of the organic layer surface and the structure is encapsulated using a glass cover attached by a bead of epoxy adhesive [EPO-TEK(302-3M)]. All the process is carried out in an inert atmosphere glovebox $(<0.1 \mathrm{ppm}$ $\mathrm{O}_{2}$ and $\mathrm{H}_{2} \mathrm{O}$ ).

\subsection{Optical measurements}

Raman measurements at room temperature were obtained collecting the light through an Olympus microscope (objectives $\times 20$ and $\times 100$ ) and a Jobin-Yvon HR 460 monochromator coupled to a $\mathrm{N}_{2}$ cooled CCD. The $647 \mathrm{~nm}$ line of an $\mathrm{Ar}-\mathrm{Kr}$ laser was used in Raman measurements with a Kaiser Super-Notch-Plus filter to suppress the elastic scattered light. The incident power was reduced to few $\mathrm{mW}$ in order to avoid local heating of the samples. The electroluminescence $(\mathrm{EL})$ spectra, luminance, spectral radiance and the Commission Internationale de L'Eclairage coordinates (CIE coordinates) from diodes were recorded with a Konica-Minolta CS-2000 spectroradiometer in the same excitation conditions as those used to measure the $\mathrm{I}-\mathrm{V}$ characteristics.

\subsection{Electrical measurements}

The device I-V characteristics were measured using a semiconductor parameter analyzer Agilent $4155 \mathrm{C}$ and a SMU pulse generator Agilent 41501B. A pulse train was used as input signal. The duty cycle was set to be $0.2 \%$, with a pulse width of $0.5 \mathrm{~ms}$ for a period of $100 \mathrm{~ms}$. Refresh time between two consecutive pulses ensures long time operation without significant device degradation. Furthermore, the I-V curve stability was achieved by gradually increasing the pulse amplitude up to the point where reproducible measurements were observed. The voltage range shown is below the onset for the electroluminescence (EL).

\section{Results and discussion}

A detailed study [4] of the material optical response, that includes absorption, emission and time resolved emission, and electrochemical characterization in diluted solution, revealed that neither the photophysical properties not electrochemical properties of the copolymers in diluted solution are affected by the introduction of the naphthalimide chromophore. Minimal interaction between chromophores (PFP and naphthalimide) in the ground state of the resulting copolymers were observed in absorption spectra and the same blue emission of PFP at $420 \mathrm{~nm}$ plus emission associated with naphthalimide pendant group peaking at $622 \mathrm{~nm}$ were observed. However, emission of P1-P4 copolymers thin films is dominated by an orange broad band $(500-650 \mathrm{~nm})$ centered at $530 \mathrm{~nm}$ and the blue PFP-related band ( $420 \mathrm{~nm}$ ) almost vanishes when increasing the naphthalimide content. This orange band arises from a center associated to the naphtalimide chromophore significantly enhanced by molecular interactions due to increased energy transfer efficiency in solid state compared to solutions.

In order to check if the naphthalimide content in the copolymer induces conformational or morphological changes in solid state that could influence the transport properties, micro-Raman spectra recorded at $647 \mathrm{~nm}$ excitation wavelength from the pristine materials (powder) have been recorded and are shown in Fig. 2. No significant changes in the vibrational modes and no new modes comparing to PFP spectrum, that could be indicative of conformational or morphological changes, are detected. Modes associated with the naphthalimide pendant group are not detected at the concentrations of our compounds. The assignment of the main vibrational peaks of the PFP pristine material due to $C=C$ vinyl group stretching, the $\mathrm{C}=\mathrm{C}$ phenyl stretching and the backbone $\mathrm{C}-\mathrm{C}$ stretch modes are indicated in the figure $[8,9]$. Thus, the naphthalimide content does not introduce conformational changes in the co-polymers at the concentration used.

EL spectra of the devices fabricated with structure ITO/PEDOT/PSS/active layer/Ba/Al using P1 $(x=0.0005), \quad$ P2 $(x=0.005), \mathbf{P 3}(x=0.05)$ and $\mathbf{P 4}(x=0.08)$ as active layers are shown in Figs. 3 and 4 . The P1 based OLED, exhibits the blue band $(418 \mathrm{~nm})$ related to PFP $(x=0)$ and the broad orange band (centered at $520 \mathrm{~nm}$ ) associated with naphthalimide in solid state [6], resulting in white emission (see $\operatorname{CIE}(x, y)$ diagram in inset of Fig. 3a). The 418-band to 520-band intensity ratio evolves with driven current resulting in almost pure white color $(0.26,0.30)$ for low currents to stable cool white $(0.21,0.23)$ as we can observe in 


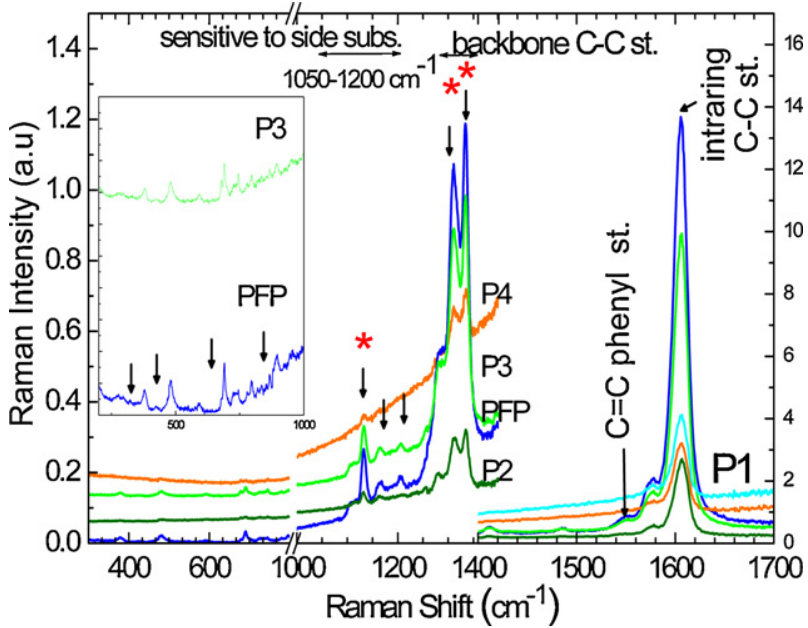

Fig. 2. Raman spectra of the copolymers as-synthesized (powder) recorded at room temperature, $\lambda_{\text {exc }}=647 \mathrm{~nm}$ ( spot size around $5 \mu \mathrm{m}$ ).

CIE diagram and Fig. 3b. The device exhibits a maximum luminous efficiency of $9.42 \mathrm{Cd} / \mathrm{A}$ at $50 \mu \mathrm{A}$ and $7.5 \mathrm{~V}$ with a brightness of $102 \mathrm{Cd} / \mathrm{m}^{2}$.

The EL emission of the P2, P3 and P4 based devices (Fig. 4) is dominated by the naphthalimide contribution that red-shifts with increasing chromophore concentration, peaking at $531 \mathrm{~nm}$ (P2), $536 \mathrm{~nm}$ (P3) and $552 \mathrm{~nm}$ (P4). Only for P2 is observed a noticeable contribution of the blue band associated with PFP. The emission color is quite stable in these devices as can be observed in the CIE diagrams. The slight variation in the blue/red emission ratio observed in this sample with increasing current injection is attributed to a saturation of the blue-to-red energy transfer rate, due to the small amount of naphtalimide concentration. In P2, P3 and P4 samples, with a higher chromophore concentration, the orange contribution associated to naphtalimide dominates and the emission is very stable with applied voltage. The maximum luminous efficiencies are 3.0, 19.5 and $6.7 \mathrm{Cd} / \mathrm{A}$ for P2, P3 and P4 respectively at driving currents of $73 \mu \mathrm{A}, 39 \mu \mathrm{A}$ and $400 \mu \mathrm{A}(14 \mathrm{~V}$,

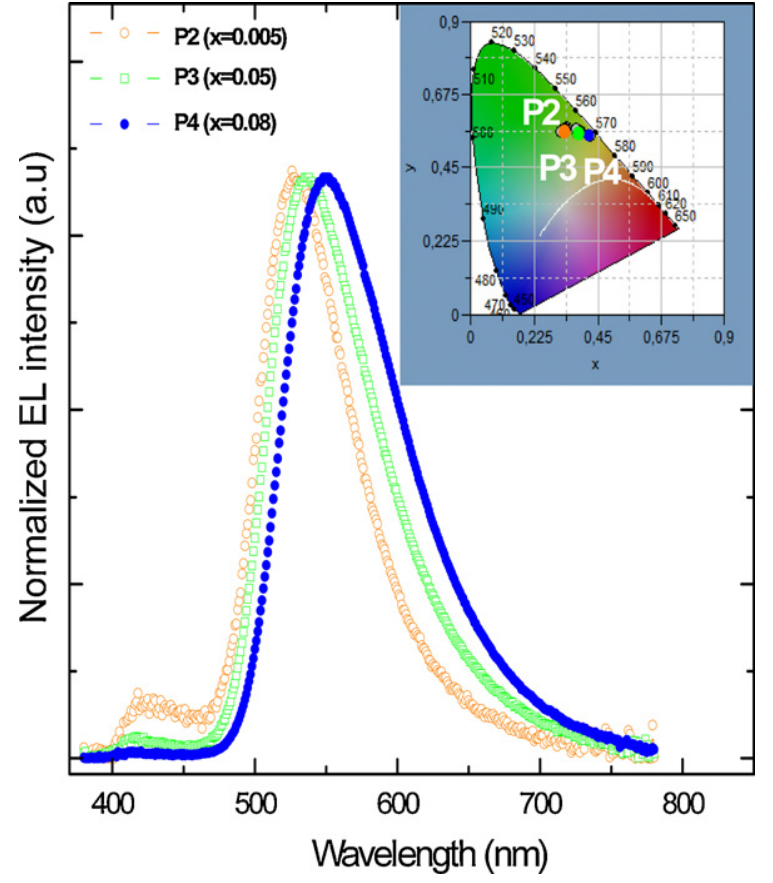

Fig. 4. Normalized EL spectra of the P2, P3 and P4 based devices and. CIE coordinates are shown in the inset.

$14 \mathrm{~V}$ and $11 \mathrm{~V}$ ) and brightness of 72,130 and $850 \mathrm{Cd} / \mathrm{m}^{2}$. The lowest value corresponds with the lowest PL quantum yield obtained for P2 thin film [6]. Fig. 5 shows the luminous efficiency dependence on the driving current of the devices. The luminous efficiency of P4-based OLED shows a continuous increase with driving current. We remark the low driving currents $(\mu \mathrm{A})$ in these solution processed single layers devices, allowing to be controlled by organic field effect transistors (OFETs).

In order to investigate how the introduction of the naphthalimide in different concentrations affects the conductivity properties of the final co-polymers, namely carrier mobilities, the I-V characteristics have been recorded. In Fig. 6 experimental I-V curves (symbol

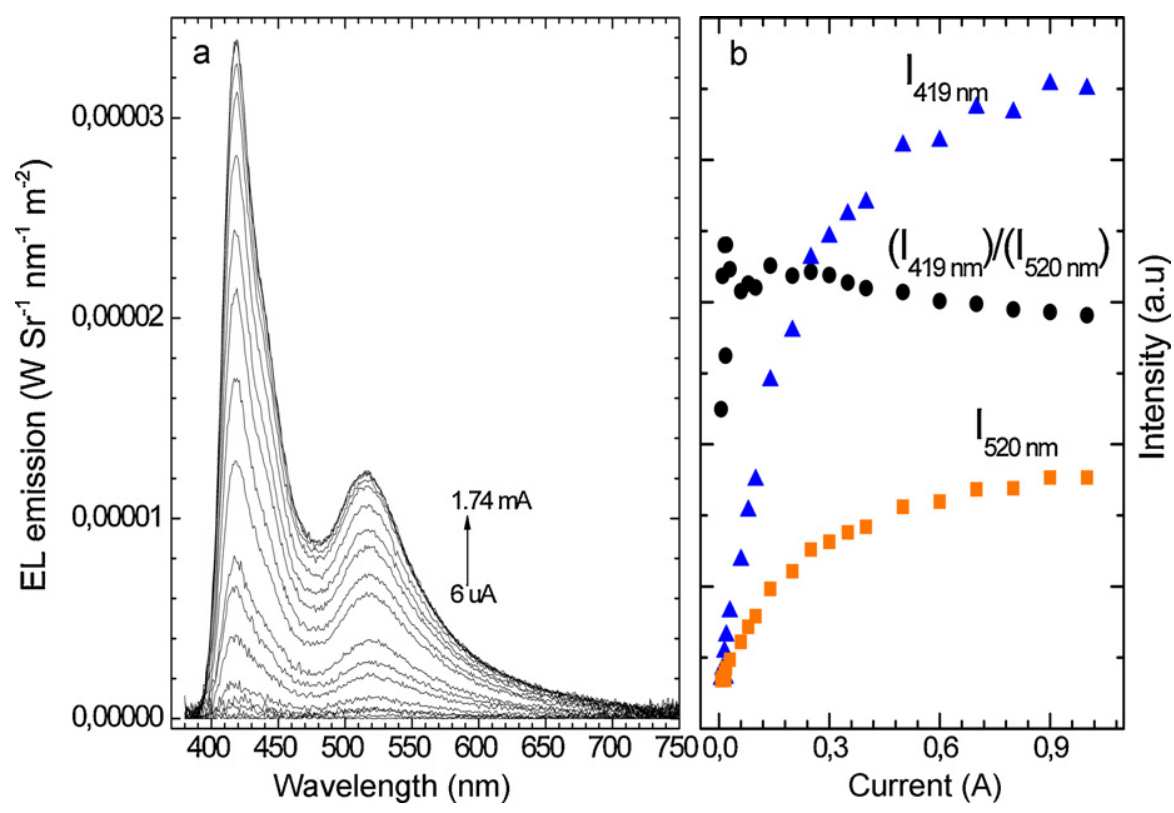

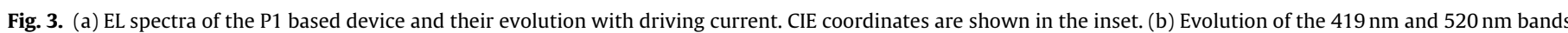
with current in the device. 


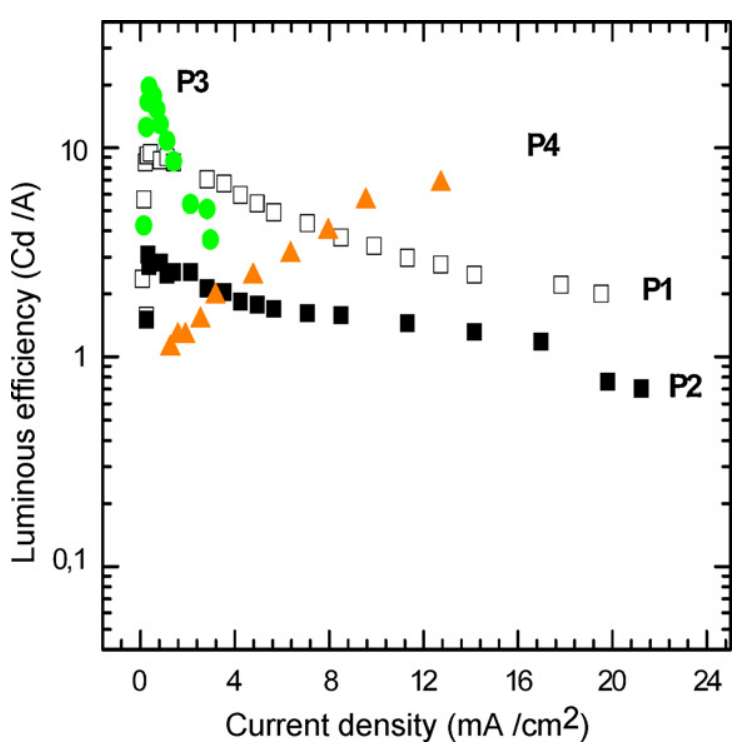

Fig. 5. Luminance efficiency dependence on the current density of the devices.

lines) of diodes based on P1, P2, P3 and P4 materials, with active layer thicknesses of $78 \mathrm{~nm}, 163 \mathrm{~nm}, 99 \mathrm{~nm}$ and $121 \mathrm{~nm}$ respectively, are shown. In all cases the I-V are plotted up to a current slightly above the onset for EL. Solid lines represent the theoretical fit to a model explained further down.

We can deduce that the introduction of the naphthalimide chromophore has a significant effect on the I-V response, however, this effect is obscured by the layer thicknesses dispersion. Layer thickness determines de internal electric field that affects bulk and injection current which determines electrical OLED response in any conduction regime, bulk or injection limited [10]. Thus the use of a conduction model is required to interpretate the $\mathrm{I}-\mathrm{V}$ response. We have chosen a single-carrier, numerical model that includes a fielddependent carrier mobility, and the presence of an electric field at

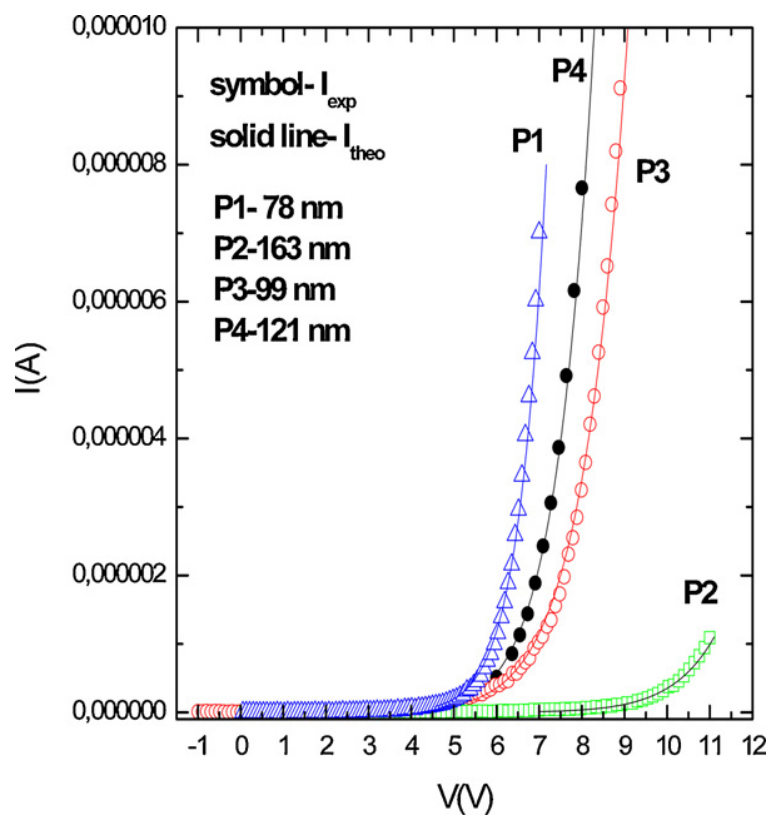

Fig. 6. Experimental $I-V$ curves of the OLED based on ITO/PEDOT:PSS/active layer/Ba/Al (symbol) and theoretical fill to a model described in the text (solid lines). The thickness of the active layers for each device is also showed. the injection interface [11]. The carrier mobility is considered upon the Murgatroyd classical expression [12,11],

$\mu(E)=\mu_{0} \theta \exp \left(\sqrt{E / E_{f}}\right)=\mu^{*} \exp (k \sqrt{E})$

where $\mu_{0}$ is the mobility at zero field, $\theta$ accounts for the fraction of injected carriers that should contribute to transport in the absence of field, and $k=\left(E_{f}\right)^{-1 / 2}$ is a phenomenological factor related to the lowering of the hoping barrier in the presence of an electric field, caused by Frenkel effect [13]. We use a quasi-Newton algorithm to fit the experimental data, considering $\mu_{0}$ and $k$ as the only physical parameters in the classical expression of mobility. Details of the model can be seen elsewhere [14,15]. In view of the energetic level diagram for both materials [4], it is expected that conduction is mainly carried out by electrons. We have been careful to use the experimental I-V interval below the onset for EL.

The $k$ values obtained are $1.99 \times 10^{-3}, 2.32 \times 10^{-3}, 1.48 \times 10^{-3}$, $1.9 \times 10^{-3}(\mathrm{~V} / \mathrm{m})^{-1 / 2}$ for $\mathbf{P 1}, \mathbf{P 2}, \mathbf{P 3}$ and $\mathbf{P 4}$ respectively. The $k$ value obtained for similar OLEDs based on a commercial polyfluorene, poly(9,9-di-n-octylfluorenyl-2,7-diyl) (PFO), is $k_{\mathrm{PFO}} \approx 3 \times 10^{-3}(\mathrm{~V} / \mathrm{m})^{-1 / 2}$. Such a lower $k$ in our materials leads to a noticeable increase of the threshold voltage (several volts) for a similar structure, as we have obtained. The higher threshold voltage for P2-based device corresponds with the thicker active layer, $163 \mathrm{~nm}$ (Fig. 6).

With respect to $\mu^{*}$ values, the resulted values are $7.5 \times 10^{-14}$, $1.66 \times 10^{-14}, 1.2 \times 10^{-11}$ and $2.91 \times 10^{-12}\left(\mathrm{~m}^{2} / \mathrm{Vs}\right)$ for P1, P2, P3 and $\mathbf{P} \mathbf{4}$ respectively. These small values could be explained by a very low $\theta$ factor. Although this is not a direct measurement of mobility, which may be more accurately determined by time-of-flight or by admittance measurements, this method provides a quick estimation which allows to observe a rough increase in the electron mobility values with concentration of the chromophore. Several authors [16] pointed out that dealing with a hoping-based transport, the density of localized states (site density) is one of the main factors involved in the carrier mobility. Thus, an increase of the localized states derived by a higher chromophore concentration would to imply that the electron mobility is favored.

\section{Conclusions}

White emission with high luminous efficiency of $9.42 \mathrm{Cd} / \mathrm{A}$ is obtained for the $x=0.0005 \%$ co-polymer (where $x$ means the fraction of monomers with naphtalimide pendant groups) at very low driving currents $(47 \mu \mathrm{A})$ in a single-layer, single-component WPLED fabricated by solution methods. It makes these materials promising candidates for being integrated in flat panel displays.

\section{Acknowledgments}

Financial support by Comunidad Autónoma de Madrid under project S2009/MAT-1756, S2009/MAT-1467 and by Ministerio de Educación y Ciencia (Spain) under MAT2009-08786, CTQ2010-14982 and TEC2009-13991-C02-02 projects is gratefully acknowledged. Also, we thank to UCM-BSCH join project GR35/10A-910759.

\section{References}

[1] http://spectrum.ieee.org/static/special-report-top-11-technologies-of-thedecade.

[2] http://www.osram-os.com/osram_os/EN/Press/Press_Releases/Organic_LED/ index.html.

[3] Current Organic Light Emitting Diode Projects in the U.S. Department of Energy, http://www1.eere.energy.gov/buildings/ssl/organic/current_organic.html.

[4] T.K. Hatwar, J.P. Spindler, M.L. Ricks, R.H. Young, Y. Hamada, N. Saito, K. Mameno, R. Nishikawa, H. Takahashi, G. Rajeswaran, Proceedings of SPIE, vol. 5214, SPIE, Bellingham, WA, 2004. 
[5] L. Liu, F. Chen, B, Xu, Y, Dong, Z Zhao, Synth, Met, 160 (17-18) (2010) 1968-1972.

[6] C. Coya, R. Blanco, R. Juárez, R. Gómez, R. Martínez, A. de Andrés, A.L. Álvarez, C. Zaldo, M.M. Ramos, A. de la Peña, C. Seoane, J.L. Segura, Eur. Polym. J. 46 (8) (2010) 1778-1789.

[7] C. Coya, A. de Andrés, R. Gómez, C. Seoane, J.L. Segura, J. Lumin. 128 (2008) 761

[8] R. Aroca, Surface-Enhanced Vibrational Spectroscopy, John Wiley and Sons, 2006.

[9] M. Ali Ifttekhar Arif, Raman Scattering Studies and charge transport in polyfluorenes, Thesis dissertation, 2007.
10] V.I. Arkhipov, EV. Emelianova, Y.H. Tak, H. Bassler J. Appl Phys. 84(1998) 848.

[11] J. Bisquert, J.M. Montero, H.J. Bolink, E.M. Barea, G. García-Belmonte, Phys. Status Solidi A 203 (2006) 3762.

12] H. Bässler, Phys. Status Solidi B 175 (13) (1993).

[13] P.N. Murgatroyd, J. Phys. D: Appl. Phys. 3 (1970) 151-156.

[14] C. Coya, A.L. Alvarez, W.S. Yoon, S.Y. Park, J. Appl. Phys. 109 (2011) 094507.

[15] A.L. Alvarez, B. Romero, B. Arredondo, X. Quintana, R. Mallavia, J.M. Oton, Int. ] Numer. Model. 23 (4-5) (2010) 332.

[16] S. Baranovski, Charge Transport in Disordered Solids with Applications in Electronics, Wiley, 2006. 